\title{
Dynamical patterns in nematic active matter on a sphere
}

\author{
Silke Henkes, ${ }^{1, *}$ M. Cristina Marchetti, ${ }^{2, \dagger}$ and Rastko Sknepnek ${ }^{3, \dagger}$ \\ ${ }^{1}$ Institute for Complex Systems and Mathematical Biology, Department of Physics, University of Aberdeen, \\ Aberdeen AB24 3UE, United Kingdom \\ ${ }^{2}$ Department of Physics and Soft Matter Program, Syracuse University, Syracuse, New York 13244, USA \\ ${ }^{3}$ School of Sciences and Engineering and School of Life Sciences, University of Dundee, Dundee DD1 4HN, United Kingdom
}

(Received 16 May 2017; published 16 April 2018)

\begin{abstract}
Using simulations of self-propelled agents with short-range repulsion and nematic alignment, we explore the dynamical phases of a dense active nematic confined to the surface of a sphere. We map the nonequilibrium phase diagram as a function of curvature, alignment strength, and activity. Our model reproduces several phases seen in recent experiments on active microtubule bundles confined the surfaces of vesicles. At low driving, we recover the equilibrium nematic ground state with four $+1 / 2$ defects. As the driving is increased, geodesic forces drive the transition to a polar band wrapping around an equator, with large empty spherical caps corresponding to two +1 defects at the poles. Upon further increasing activity, the bands fold onto themselves, and the system eventually transitions to a turbulent state marked by the proliferation of pairs of topological defects. We highlight the key role of the nematic persistence length in controlling pattern formation in these confined systems with positive Gaussian curvature.
\end{abstract}

DOI: 10.1103/PhysRevE.97.042605

\section{INTRODUCTION}

Active matter consists of self-driven agents that individually dissipate energy and organize in collective self-sustained motion. Active systems are maintained out of equilibrium by a drive that acts independently on each agent, breaking time-reversal symmetry locally, rather than globally as in more familiar condensed matter systems driven out of equilibrium by external fields or boundary forces. Realizations span many scales, both in the living and nonliving world, from bird flocks to bacterial suspensions, epithelial cell layers, and synthetic microswimmers [1,2].

Active particles are often elongated and form active liquid crystal phases, with either polar or nematic symmetry, and emergent patterns controlled by the interplay of orientational order and active flows. Novel effects predicted and observed in simulations and experiments include long-range order in two dimensions [3], spontaneous laminar flow [4], giant number fluctuations [5-7], novel rheology [8,9], and active turbulence accompanied by the proliferation of topological defects that drive the self-sustained dynamics $[10,11]$. Very recently, it was shown that monolayers of various cell types at confluence, where there are no gaps between cells, exhibit some degree of nematic order [12-14]. Topological defects were found to affect the location of cell death and extrusion sites in MDCK monolayers [14] and neural progenitor cell cultures [13]. Much of this rich behavior is captured well by active hydrodynamics.

More surprises arise when active systems are confined by bounding surfaces or to geometries that do not allow defect-

\footnotetext{
*shenkes@abdn.ac.uk

†mcmarche@syr.edu

${ }^{\dagger}$ r.sknepnek@dundee.ac.uk
}

free ground-state configurations. When confined to a box, active particles accumulate at the boundary, with the strongest accumulation at corners, demonstrating the dramatic effects of wall curvature $[15,16]$. When constrained to move on curved surfaces, as realized for instance when cells migrate in the gut epithelium [17] or on the surface of the growing cornea [18], the interplay of activity and curvature can drive novel dynamical structures. Keber et al. [19] have studied this interplay under controlled conditions in active vesicles obtained by confining an active nematic suspension of kinesin-microtubule bundles to the surface of a lipid vesicle. Topological defects are unavoidable even in the ground state when a nematic is confined to the surface of a sphere, where the net topological charge must be 2, i.e., equal to the Euler characteristic of the two-sphere. In equilibrium, the lowest-energy configuration consists of four $+1 / 2$ disclinations arranged at the corner of a tetrahedron inscribed in the sphere [21,22]. In the active vesicles of Ref. [19], this four-defect configuration oscillates at a well-defined rate controlled by the concentration of ATP between the tetrahedral configuration and a planar one, with the four defects equally spaced on an equator. A minimal model of $+1 / 2$ active defects as self-propelled particles [23], subsequent hydrodynamic descriptions [24], and a particle-based extensile nematic simulation [25] reproduce the oscillatory behavior, but are inadequate to describe the rich succession of dynamical states observed when decreasing the size of the vesicles, including defect-driven protrusions, and two +1 aster defects at the poles with spontaneously folding nematic bands.

In this paper, we examine the rich dynamics of a dry active nematic confined to the surface of a two-sphere by considering a model of soft spherical self-propelled agents with short-range repulsion and nematic alignment. Despite using isotropic agents and omitting effects of the surrounding fluid flow, the model reproduces a number of the experimentally 

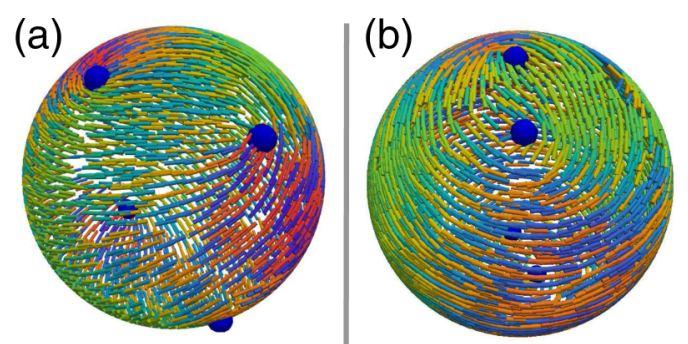

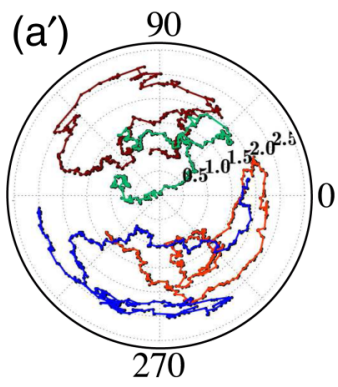

$4+1 / 2$ defects

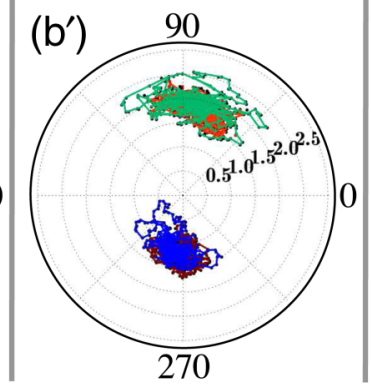

merging

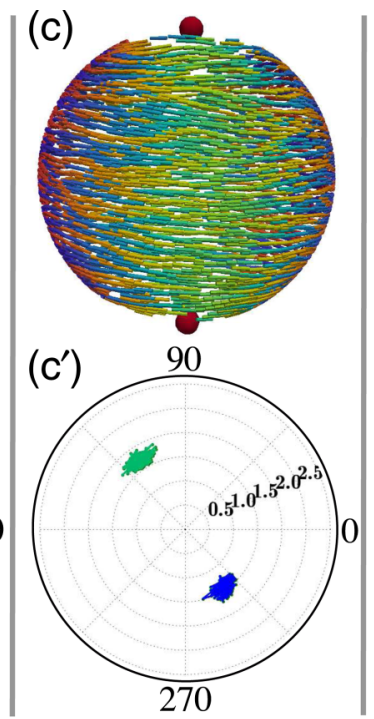

$2+1$ defects: band

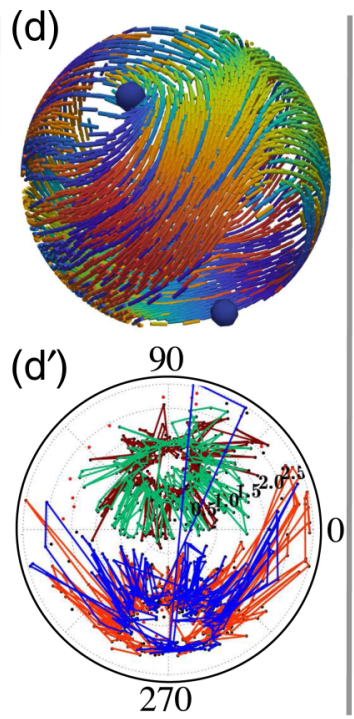

bending

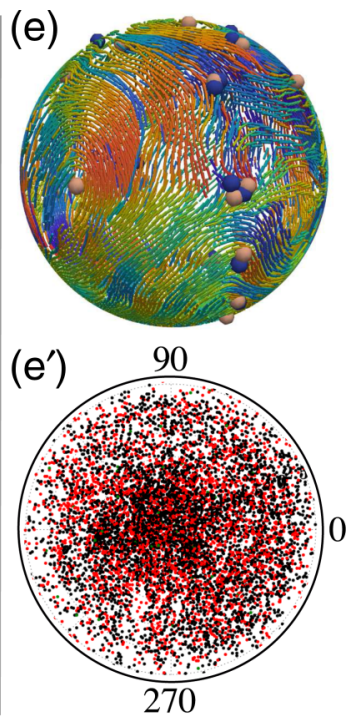

active turbulence

FIG. 1. Active nematic patterns formed by agents self-propelled at speed $v_{0}$ on a sphere of radius $R$. Individual agents randomly reverse their direction of self-propulsion at rate $\tau_{\text {flip }}^{-1}$ and align nematically with neighbors. The strength of the nematic alignment is controlled by the coupling constant $J$. Activity (quantified by $v_{0}$ ) increases from left to right (see also movies M1-M5 in the Supplemental Material [20]). Top row: Nematic patterns and defect positions. Individual agents are represented by bars, colored by the $x$ component of their director. Defects are represented by dots, colored by charge (dark blue: $+1 / 2$, tan: $-1 / 2$, red: +1 ). Polarization sorting and the formation of lanes traveling in opposite directions is evident in (d) and (e). Bottom row: Stereographic projections of defect trajectories in the corresponding states (see also Fig. 2 for the corresponding tracks). Here $+1 / 2$ and $-1 / 2$ defects are represented by black and red (grey) dots, +1 defects are green (grey) dots, and where possible, we have tracked defect trajectories in different colours. All images are for $\tau_{\text {flip }}=100$ and $R=30$, except for (e) where $R=50$. (a), ( (a') Four $+1 / 2$ defects for $v_{0}=0.4$ and $J=1$; (b), ( ( $\left.\mathrm{b}^{\prime}\right)$ merging defect state for $v_{0}=1, J=5(\mathrm{~b})$ and $v_{0}=1.2$ and $J=2\left(\mathrm{~b}^{\prime}\right)$;

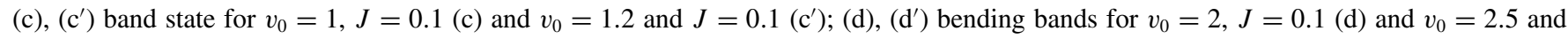
$J=0.1\left(\mathrm{~d}^{\prime}\right) ;(\mathrm{e}),\left(\mathrm{e}^{\prime}\right)$ active turbulence for $v_{0}=4, J=1(\mathrm{e})$ and $v_{0}=5$ and $J=5\left(\mathrm{e}^{\prime}\right)$.

observed dynamical structures [19], including the oscillating state of four $+1 / 2$ disclination defects, an equatorial nematic band and a state where the band folds onto itself. Furthermore, for large spheres our model predicts a transition to active turbulence with proliferation on unbound defect pairs, a state not reported in the experiments of Keber et al. probably due to the small size of the vesicles. The rich succession of states obtained with increasing activity is shown in Figs. 1 and 2. The fact that a dry-agent-based model without any flow reproduces most of the dynamical states observed in a complex experimental system suggests that these emergent structures are generic for active nematics on curved surfaces, independent of microscopic details. This rich dynamical behavior is controlled by the interplay of curvature, long-range nematic order and activity, and much remains to be explored. Recently, nematic order has been reported in planar monolayer of motile cells studied in vitro. Since the growth of curved tissue sheets is a central part of development and organogenesis, the findings reported here could also have a broad impact in understanding biophysical processes in developmental biology.

Circulating band states have also been reported by two of us in a simulation of soft self-propelled agents on a sphere, but with polar alignment [26]. Recent work by one of us has additionally demonstrated that band formation on a sphere is a universal feature of the Toner-Tu equations for polar flocks [27]. It was also recently shown that on ellipsoids, the band localizes to the low-curvature region [28]. Here we show that active nematics also exhibit band states arising from the interplay of active motion and curvature. Our work shows that the formation of the band state is a generic property of active systems, independent of their symmetry and that it arises from the intrinsic tendency of active particles to move along geodesic lines on curved surfaces. The identification of this key mechanism for driving pattern formation on curved topologies is an important result of our work.

A second, more subtle, finding concerns the emergence of hydrodynamics from agent-based models. Derivations of hydrodynamic equations from microscopic dynamics can be carried out at low density [1], but become challenging in the dense limit [29]. In particular, although our system behaves like a nematic fluid at long times and large length scales, it also exhibits a substantial amount of short-range polar order and local flocking at intermediate time scales, suggesting that a suitable continuum model may require inclusion of a polarization field in conjunction to a nematic order parameter. A similar result was reported for a related model in the plane [30], hence is not the result of curvature. This behavior is also reminiscent of that of collections of self-propelled rods [31] - polar active agents that exhibit nematic order at large scales. This local polar order becomes important in confined geometries such as a sphere when the persistence length of the motion becomes comparable to the sphere radius and drives polarity sorting and leads to the folding band regime shown in Fig. 9. In the experiments of Ref. [19] similar structures occur when the length of the microtubule bundles is comparable to the size of the vesicle. 

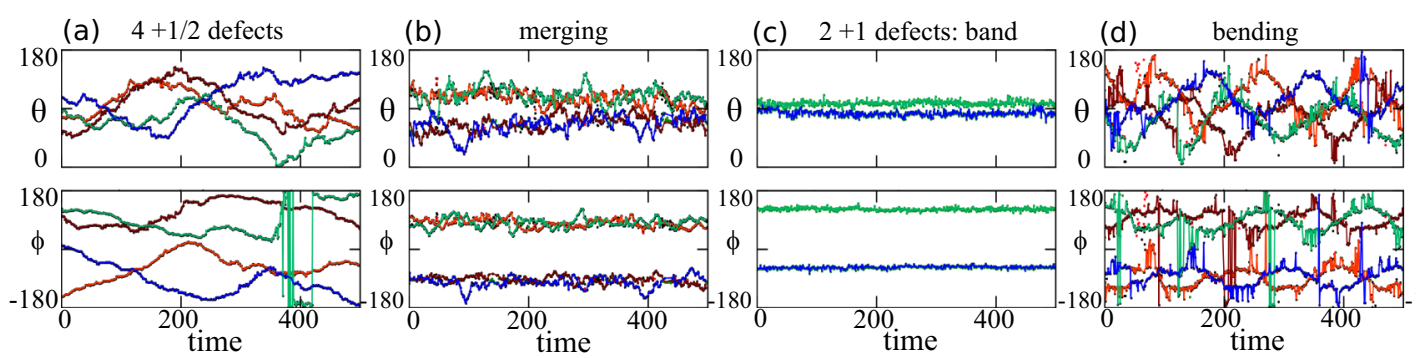

(e) nematic turbulence

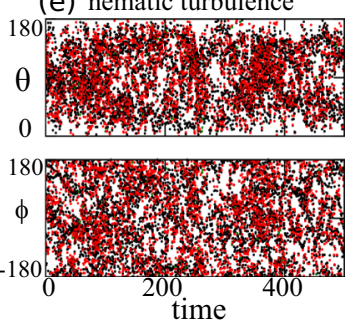

FIG. 2. Defect tracks as a function of time for the same simulation runs as the stereographic projections in Fig. 1. (a) Four defects for $v_{0}=0.4$ and $J=1$. (b) Merging defects for $v_{0}=1.2$ and $J=2$. (c) Band state for $v_{0}=1.2$ and $J=0.1$. (d) Bending bands for $v_{0}=2.5$ and $J=0.1$. (e) Active turbulence for $v_{0}=5$ and $J=5$.

The paper is organized as follows. In Sec. II we introduce the model of soft active agents moving on the surface of a twosphere. In Sec. III, we outline methods used to analyze behavior of the system, followed by a detailed characterization of the five distinct dynamical patterns that we have observed presented in Sec. IV. Finally, we conclude in Sec. V. In Appendix A we introduce the algorithms used for identifying and tracking defects.

\section{MODEL: SOFT ACTIVE AGENTS ON A SPHERE}

Active particles move through a medium that mediates long-range hydrodynamic interactions. Here we consider the dry limit, where the damping from the medium dominates over hydrodynamic interactions and the fluid only provides singleparticle friction. Our system consists of $N$ self-propelled soft spherical agents of radius $\sigma$ confined to move on the surface of a two-sphere of radius $R$ (Fig. 3). Each agent is characterized by its position $\mathbf{r}_{i}$ and a unit vector $\mathbf{n}_{i}$ or director denoting the direction of self-propulsion. Although confinement to the surface of a sphere implies that agent positions are parametrized by just two coordinates, e.g., azimuthal and polar angles, in numerical simulations it is more convenient to work in threedimensional Euclidean space and explicitly impose the sphere

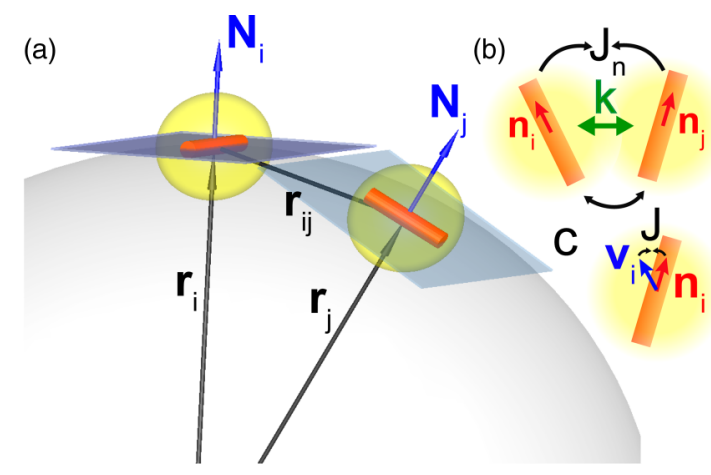

FIG. 3. (a) Self-propelled agents are constrained to the surface of a sphere of radius $R$. Agents have an internal degree of freedom, the director $\mathbf{n}_{i}$ (red/dark cylinders). The position of each agent is given by $\mathbf{r}_{i}$, measured from the center of the sphere. Distances between agents, $r_{i j}=\left|\mathbf{r}_{i}-\mathbf{r}_{j}\right|$, are calculated as the Euclidean distance in $\mathbb{R}^{3}$ space. (b) Agents are subject to a soft-core repulsion and experience torque which nematically aligns them to their immediate neighbors. (c) Additionally, each agent has a preference to align its director to the direction of its motion. constraint at each time step. This is accomplished through projection operators. The coupled equations for translational and rotational motion are then given by

$$
\begin{aligned}
\dot{\mathbf{r}}_{i}=\mathbf{P}_{i}^{T}\left[v_{0} \kappa_{i}(t) \mathbf{n}_{i}+\mu \sum_{j} \mathbf{F}_{i j}\right], \\
\dot{\mathbf{n}}_{i}=\mathrm{P}_{i}^{N}\left[-2 J_{n} \sum_{j}\left(\mathbf{n}_{i} \cdot \mathbf{n}_{j}\right)\left(\mathbf{n}_{i} \times \mathbf{n}_{j}\right)\right. \\
\left.-2 J_{v}\left(\mathbf{v}_{i} \cdot \mathbf{n}_{i}\right)\left(\mathbf{v}_{i} \times \mathbf{n}_{i}\right)\right]\left(\hat{\mathbf{N}}_{i} \times \mathbf{n}_{i}\right),
\end{aligned}
$$

where $\mathbf{P}_{i}^{T}[\mathbf{a}]=\mathbf{a}-\left(\hat{\mathbf{N}}_{i} \cdot \mathbf{a}\right) \hat{\mathbf{N}}_{i}$ and $\mathrm{P}_{i}^{N}[\mathbf{a}]=\left(\mathbf{a} \cdot \hat{\mathbf{N}}_{i}\right)$ project any three-dimensional vector $\mathbf{a}$ onto the tangent plane and the surface normal to the sphere, respectively, with the local surface normal given by $\hat{\mathbf{N}}_{i}=\mathbf{r}_{i} / r_{i}$ [26].

The first term on the right-hand side of Eq. (1a) describes self-propulsion at speed $v_{0}$. The nematic symmetry is implemented by reversing the direction of self-propulsion at time intervals drawn from a Poisson distribution of mean $1 / \tau_{\text {flip }}$ through a zero average bimodal noise $\kappa_{i}(t)= \pm 1$ [7]. The second term in Eq. (1a) describes soft pairwise repulsive forces of stiffness $k, \mathbf{F}_{i j}=-k\left(2 \sigma-r_{i j}\right) \frac{\mathbf{r}_{i j}}{r_{i j}}$, with $\mathbf{r}_{i j}=\mathbf{r}_{j}-\mathbf{r}_{i}$ and $r_{i j}=\left|\mathbf{r}_{i j}\right|$, for $r_{i j}<2 \sigma$ and $\mathbf{F}_{i j}=0$ otherwise [Fig. 3(b)], and $\mu$ the mobility. Here $r_{i j}$ is the Euclidean distance computed in $\mathbb{R}^{3}$. The rotational dynamics of $\mathbf{n}_{i}$ is governed by Eq. (1b). It contains two contributions to the torque: (i) nematic alignment of neighboring directors at rate $J_{n}$, with the sum extending over all neighbors within a radius $r_{c}=2.4 \sigma$; and (ii) alignment of the director $\mathbf{n}_{i}$ with the direction of its own velocity, $\mathbf{v}_{i}=\dot{\mathbf{r}}_{i}$, at rate $J_{v}$.

In the absence of interactions, the mean-square displacement of a single agent is diffusive at long times with effective diffusion constant $D_{0}=v_{0}^{2} \tau_{\text {flip }} / 4$. The random flips serve the same function as rotational noise in active Brownian particle models [32] and we therefore neglect rotational noise in Eq. (1b). Additionally we neglect Brownian noise in the translational dynamics because we focus on the high-density regime of a sphere with packing fraction $\phi=N \sigma^{2} / 4 R^{2}=1$, where the dynamics is dominated by steric repulsion and noise is negligible compared to the effects of collisions.

To make contact with more familiar forms of the equations of motion for active nematic agents, we note that in the local 
tangent plane at position $\mathbf{r}_{i}$ we can write $\mathbf{n}_{i}=\left(\cos \theta_{i}, \sin \theta_{i}\right)$, where $\theta_{i}$ is the angle with the local $x$ axis. Then the $J_{n}$ term yields a torque proportional to $\sin 2\left(\theta_{j}-\theta_{i}\right)$ (up to correction terms of order $2 \sigma / R$ due to parallel transport), which is the nematic coupling used in previous literature [7]. In the same coordinate system the second term on the right-hand side in Eq. (1b) takes the form $J_{v}\left|\mathbf{v}_{i}\right|^{2} \sin 2\left(\theta_{i}^{v}-\theta_{i}\right)$, where $\theta_{i}^{v}$ is the angle of the velocity vector with the local $x$ axis in the tangent plane, hence it tends to align the director with the particle's velocity $[33,34]$. This term is required to ensure torque transfer between orientational and translational degrees of freedom, since for spherical agents changes in the direction of motion are not directly coupled to the director $\mathbf{n}_{i}$. As we illustrate in Appendix E, this term is required for nucleation of defect pairs. In models with anisotropic agents, such coupling arises naturally from steric interactions that are anisotropic and transfer torque, stirring the local structure when an agent changes its direction. For simplicity, in the following we set $J_{v}=J_{n} \equiv J$.

To date, three other microscopic models of active nematics that fully include steric effects have been investigated with large-scale simulations. In planar geometry, DeCamp et al. [35] simulated a collection of elongated rodlike particles whose length grows at a steady rate, directly producing an extensile stress, until at a critical length a rod splits into two while two other rods merge to maintain number conservation. This model exhibits an active nematic state with somewhat different defect symmetries than observed in the experiment of Keber et al. [19]. Shi and Ma [30] studied a model of self-propelled ellipsoids with reversal of the direction of self propulsion similar to the one employed in the present study. They focused on the regime of very short $\tau_{\text {flip }}$ and found very weakly extensile forces in this region of parameters, together with very slowly moving $+1 / 2$ defects. As we will show below, our results confirm the observations of Shi and Ma that defects are only very weakly motile and the dynamics is dominated by strongly fluctuating locally polar flows (see Appendix C). Very recent work by Alaimo et al. [25] on spheres and ellipsoids introduces a model with the same steric components as ours, but with a directly extensile forcing instead of self-propulsion. This leads to a locally extensile material with an oscillating defect phase, but in contrast no band phase was reported.

Here we focus on reversing self-propelled systems where reversal is slow compared to the particle collision time, i.e., $\tau_{\text {flip }} \gg(\mu k)^{-1}$, where $(\mu k)^{-1}$ is the time scale of the interaction. This choice results in persistent dynamics on length scales $\xi=v_{0} \tau_{\text {flip }}$ that at large activity $v_{0}$ can become comparable to the size of the sphere. This is indeed the regime relevant to the experiments of Keber et al. [19], where kinesin motors walk persistently along the microtubule bundles that have lengths comparable to the radius of the vesicles. Finally, in the following we measure time in units of $\mu k$ and lengths in units of $\sigma$ and set $\tau_{\text {flip }}=100$ (except where stated otherwise).

\section{CHARACTERIZING DYNAMICAL PATTERNS AND DEFECT STRUCTURES}

In this section we briefly outline the methods used to analyze the results of our simulations. A summary of our method for identifying and tracking defects using a tessellation method in the presence of density fluctuations is given in Appendix A.

\section{A. Mean-square displacements}

To compute the mean-squared displacement (MSD) of the defects, we used geodesic distances, i.e., the minimal distance along the great circle connecting the two positions in question. For two arbitrary points $\mathbf{r}_{i}$ and $\mathbf{r}_{j}$ on the two-sphere, we have

$$
d_{i j}=R \arccos \left(\frac{\mathbf{r}_{i}}{R} \cdot \frac{\mathbf{r}_{j}}{R}\right) .
$$

Then the MSD is computed as

$$
\operatorname{MSD}(\delta t)=\langle d(t+\delta t) d(t)\rangle_{t, \text { defects }} .
$$

Tracking was not always successful, especially at larger $v_{0}$, and we excluded trajectories with too many outliers from the MSD computations. For smaller numbers of outliers, we employed an error-correction algorithm that interpolates the outlier position from the previous and next correctly tracked points. The scaling of the MSD as MSD $\sim t^{\alpha}$ allowed us to identify diffusive $(\alpha=1)$ and persistent $(\alpha>1)$ regimes, and to compute the diffusion coefficients of defect motion (Fig. 5).

\section{B. Pair angles}

We define the pair angle between defects located at $\mathbf{r}_{i}$ and $\mathbf{r}_{j}$ as

$$
\theta_{i j}=\arccos \left(\frac{\mathbf{r}_{i}}{R} \cdot \frac{\mathbf{r}_{j}}{R}\right) .
$$

For the tetrahedron the six pair angles are all $\theta_{i j}=109.47^{\circ}$, while in an alternative flat configuration seen in Ref. [19], four defect pairs with an angle of $90^{\circ}$ and two defect pairs with an angle of $180^{\circ}$ emerge, giving a mean pair angle $\left\langle\theta_{i j}\right\rangle=120^{\circ}$. We use both the $\theta_{i j}$ and $\left\langle\theta_{i j}\right\rangle$ to identify oscillations in Fig. 6. The +1 defects of the band state are nearly separated by $180^{\circ}$, and in the merging state, pairs of $+1 / 2$ defects approach each other. Pair defect angle distributions are shown in Figs. 7(a) and 7(c).

\section{Angular profiles}

In the band state, a density profile emerges with bald spots at the poles and greater density at the equator. In parallel, the director field shows a systematic inclination towards the equator. Both effects are due to curvature-induced forces [26]. We reoriented configurations with the pole axis along $\mathbf{z}$ as follows. We first computed an estimate $\mathbf{z}_{\text {Ine }}$ of the axis $\mathbf{z}$ from the eigenvector associated to the smallest eigenvalue of the inertial tensor $\hat{I}=\sum_{i} \mathbf{r}_{i} \otimes \mathbf{r}_{i}$. Then we determined a \pm 1 orientation for each velocity vector $\mathbf{v}_{i}$ on the basis that $\mathbf{r}_{i} \times \mathbf{v}_{i}$ is largely either parallel or antiparallel to $\mathbf{z}_{\text {Ine }}$, and finally computed

$$
\mathbf{z}=\sum_{i} \mathbf{r}_{i} \times \operatorname{orient}\left(\mathbf{v}_{i}\right)
$$

and normalized it. The density profiles in Figs. 7(b) and 7(d) and the orientation profile of the director $\alpha_{i}=\arccos \left(\mathbf{n}_{i} \cdot \mathbf{e}_{\phi}\right)$ in Fig. 7(a) have been averaged over all snapshots of several runs. 


\section{RESULTS}

We have studied spherical vesicles of radii $R=5-50$ for packing fraction $\phi=1$, corresponding to full coverage of the sphere and $10^{2}-10^{4}$ agents. Results reported here are for $R=30$, unless stated otherwise. All our simulations are in a steady state, in the sense that they have relaxed from an initial random configuration. We study both the fast dynamics that immediately follows the relaxation to the steady state by integrating Eqs. (1) for a total of $1.5 \times 10^{3}$ time units with time step $\delta t=10^{-3}$ using a standard Euler-Maruyama algorithm, and the dynamics at long times with $\delta t=0.01-0.02$ for a total integration time of up to $2 \times 10^{5}$ time units.

In Fig. 1, we show the succession of states obtained with increasing activity $v_{0}$. As required by the Poincaré-Hopf theorem [36], a nematic field on the surface of a sphere always contains topological defects with total charge 2 . This can be satisfied by two +1 defects at the poles of the sphere, or four $+1 / 2$ defects at the corners of a tetrahedron, or else by a turbulent state with many defects of both positive and negative charges, but adding up to a net charge of +2 . The tetrahedral arrangement is the ground-state configuration for a nematic liquid crystal with equal bend and splay Frank constants [21,22]. In the presence of activity the rules governing conservation of topological charge are of course unchanged, but defects often become dynamical entities that move with actively driven flows. At low activity $v_{0}$ we obtained a texture of four well-separated $+1 / 2$ disclinations, as shown in Fig. 1(a). The four defects, however, are not static, but move either diffusively or, at long times, in an oscillatory fashion. At larger values of activity, pairs of $+1 / 2$ defects are pushed toward opposite poles [Fig. 1(b)] and eventually merge resulting in a configuration of two +1 defects at the poles, with a band of nematic wrapping around a great circle [Fig. 1(c)]. Agents move within the band, with approximately equal fractions moving in clockwise and counterclockwise directions, so that nematic order is maintained with no mean flow. The bald spots at the poles can be interpreted as the cores of very large defects. The width of the band decreases when lowering the alignment coupling $J$, as observed in polar systems [26]. At even higher activity and sufficiently low alignment $J$, the band becomes unstable to bend deformations [Fig. 1(d) and Fig. 9] and one observes a complex folding dynamics, associated with sorting of particles into unidirectional lanes. Finally, upon further increase of $v_{0}$ the system exhibits turbulentlike flows with proliferation of topological defects [Fig. 1(e)], akin to that observed in planar systems.

We proceed to classify the various dynamical states depicted in Fig. 1 using the tools and methods discussed in previous section and the defect tracking algorithm outlined in Appendix A. By combining all of these results, we are able to map the complex dynamical states observed in the simulations in the phase diagram shown in Fig. 4.

\section{A. Four-defect state}

The four defects are generally motile on the sphere, as shown in Fig. 1 that displays the stereographic projections of their trajectories, with the only exception of the region of very low $v_{0}$ and $J$ where the system is effectively jammed (pale

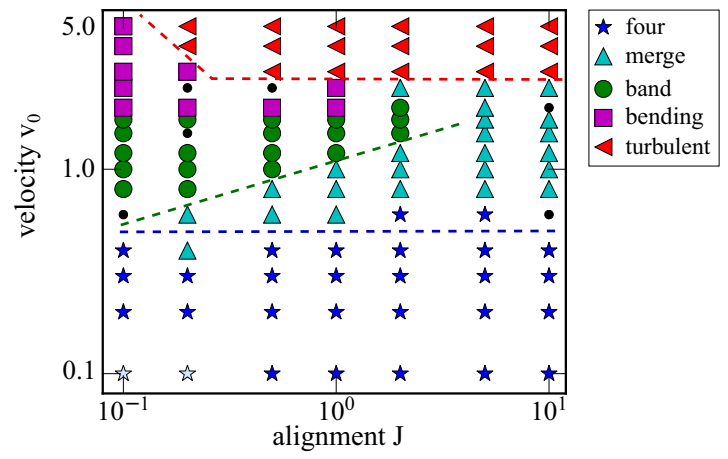

FIG. 4. Phase diagram at high $\tau_{\text {flip }}=100$ as a function of coupling $J$ and of active driving $v_{0}$. We observe a low $v_{0}$ phase with four $+1 / 2$ defects (blue stars, pale blue stars), which gives way to an intermediate set of band states with either merging defects (turquoise upright triangles) or $+1 / 2$ defects fully merged into two +1 defects at low $J$ (green circles). This phase is succeeded by turbulence above a threshold $v_{0}$ (red sideways triangles). At low $J$, a folding instability in the band shapes appears as driving increases (purple squares). Black dots denote states that we could not classify. The colored lines representing the phase boundaries are guides to the eye.

blue stars in the phase diagram Fig. 4 in a state akin to the one reported by Fily et al. [37] and by Janssen et al. [38] on the sphere ). To analyze the defect configuration and their dynamics we have examined the histogram of the angles $\theta_{i j}$ of defect pairs [Fig. 7(a)] and their mean-square displacements (Fig. 5). We uncover two distinct regimes. At intermediate times, but long after settling into a long-lived steady state, the pair angle distribution is unimodal and peaked around $\theta=109^{\circ}$ corresponding to a tetrahedral configuration. In this regime, the MSD of the defects is diffusive [Fig. 5(a)] with a diffusion coefficient that scales as $D \sim v_{0}^{2} \tau_{\text {flip }}$ as expected for particles self-propelled at speed $v_{0}$ with noisy flips of their direction of self-propulsion at rate $\tau_{\text {flip }}$. The diffusion rate also grows linearly with the alignment rate $J$, suggesting a scaling $D=v_{0}^{2} \tau_{\text {flip }} f(J \mu k)$, with $f(x) \sim x$ at small $x$. We note that in this regime the local flow and pressure fields are dominated by fluctuations (see Appendix C), and we conjecture that the diffusive defect dynamics arises because defects are carried along by the strongly fluctuating flows. With increasing activity the distribution of pair angles broadens, but remains unimodal, and the MSD begins to cross over to superdiffusive behavior at long times. Similar superdiffusive scaling was obtained by Shi and Ma for tapered rods [30] in the plane.

At very long times the defect dynamics becomes superdiffusive with a largest exponent of approximately $\alpha \approx 1.5$, and we observe oscillations akin to those reported in the experiments of Keber et al. [19], albeit only for specific parameter combinations. In Fig. 5(b), we show the MSD trajectories over the full simulation time range in such an oscillatory regime. We analyze the oscillations in Fig. 6 (see also supplemental movie M1 [20]). The top frame [Fig. 6(a), top] shows that the defect tracks perform a slow oscillatory motion that corresponds to a distinct periodic pattern of the dynamics of the six pair angles [Fig. 6(a), middle]: at regular intervals $T_{m}$, two of the pair angles reach $\theta_{i j}=180^{\circ}$, 

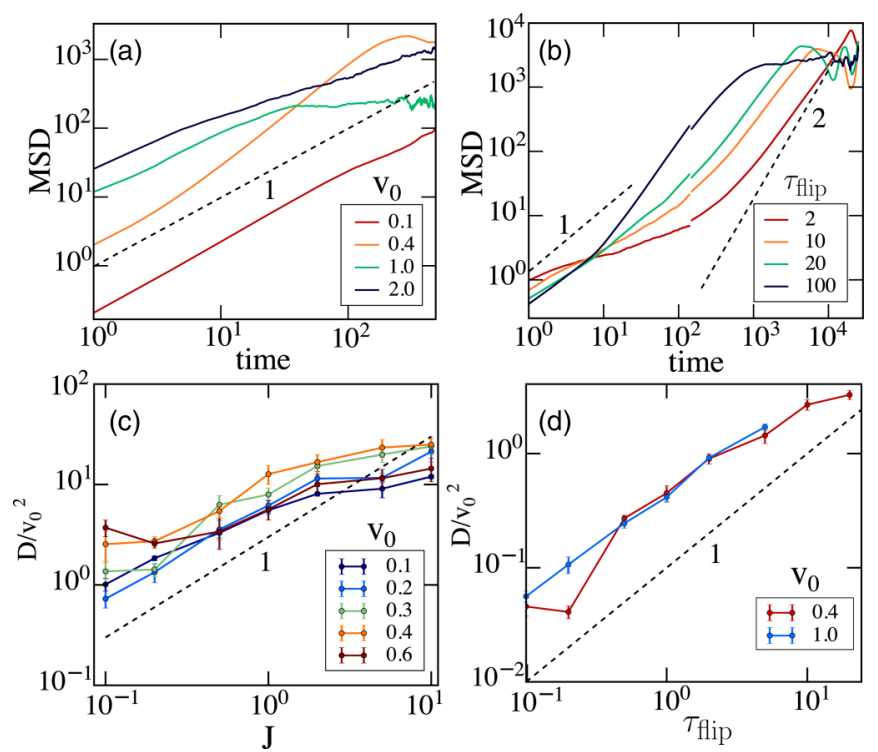

FIG. 5. Mean-square displacements (MSDs) of defects, and diffusion coefficients. (a) Four representative MSD trajectories of $+1 / 2$ defects as a function of $v_{0}$ for $J=1.0$ and $\tau_{\text {flip }}=100$. From bottom to top: diffusive motion at low $v_{0}$ becomes superdiffusive in regions with oscillations, then subdiffusive in the merging state, and close to diffusive again in the folding state. (b) The long-time MSD for oscillating systems at $J=0.1$ and $v_{0}=0.4$ becomes nearly persistent at different $\tau_{\text {flip }}$. This graph combines data from short and long simulations with fast and slow sampling, respectively, leading to the slight gap around $t=10^{2}$. Note that the gap is smaller than the statistical uncertainty (not shown for clarity). (c) Diffusion coefficient $D$ obtained from fits to the MSD, as a function of $J$ and scaled by $v_{0}^{2}$ for $\tau_{\text {flip }}=100$. (d) Scaled diffusion coefficient $D / v_{0}^{2}$ as a function of $\tau_{\text {flip. }}$.

while the remaining four angle pairs dip down to $\theta_{i j}=90^{\circ}$, corresponding to a flat defect configuration. This configuration, as reported in the experiment of Keber et al. and in analytical [24] and numerical [25] treatments, is an energy maximum reached between different tetrahedral configurations of defect positions where all $\theta_{i j}=109^{\circ}$. Different pairs traverse the flat state at each interval, with a period of two to four intervals before a recurrence. As a result, the mean pair angle $\left\langle\theta_{i j}\right\rangle$ [Fig. 6(a), bottom] oscillates with period $T_{m}$ between flat and tetrahedral configurations with $\left\langle\theta_{i j}\right\rangle=120^{\circ}$ and $\left\langle\theta_{i j}\right\rangle=109^{\circ}$, respectively. In Fig. 6(b), we show the Fourier spectrum of tracks, pair angles, and mean pair angle. The latter has a pronounced peak at $\omega_{m} \approx 2 \pi / T_{m} \approx 0.003$, as expected, and a secondary peak at $\omega_{m} / 2$. The pair angle spectrum show a superposition of frequencies in the range $\omega<0.001$, and so does the spectrum of the individual tracks. We complement this analysis with the autocorrelation function in Fig. 6(c), which shows clear oscillations with $T_{m} \approx 2000$ for the mean angle. This complex oscillation pattern is very similar to the one observed in experiments [19].

It is worth noting that we did not observe oscillations at any $J>0.1$, and we speculate that for larger $J$ the relaxation time $1 / J$ of the director field is too fast for the flow field to coherently respond to it, so that again fluctuations dominate the dynamics.
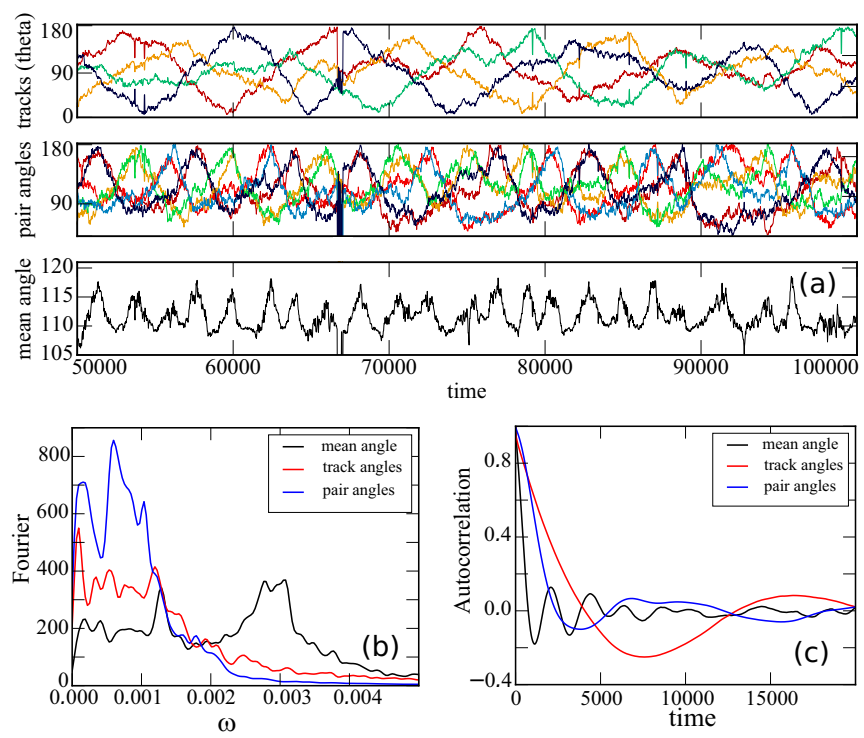

FIG. 6. Oscillations of the defects at long times, for $v_{0}=1.0$, $J=0.1$, and $\tau_{\text {flip }}=1.0$. (a) Detail of the individual defect traces (top, polar angle $\theta$ ), pair angles $\theta_{i j}$ (middle), and mean pair angle $\left\langle\theta_{i j}\right\rangle$ (bottom). Long-time track oscillations combine into a pattern where alternating pairs of angles produce a flat configuration, giving rise to 2-4 times faster mean angle oscillations. (b) Fourier spectrum of individual (red), pair (blue), and mean angles (black). The mean angle oscillation with frequency $\omega \approx 0.003$ arise from the slower oscillations of pair angles, whose spectrum exhibits several peaks at $\omega<0.001$. These curves were smoothed using convolution with a Gaussian kernel of width $2 \sigma=10^{-4}$. (c) Autocorrelation of the same, clearly showing the mean angle oscillation period $T_{m} \approx 2000$. (b) and (c) combine data from ten independent runs of length $2 \times 10^{5}$.

\section{B. Band formation and merging defects}

As $v_{0}$ increases, we observe the emergence of a nematic band wrapped around an equator that is chosen through spontaneous symmetry breaking [see Figs. 1(b) and 1(c)]. The four $+1 / 2$ defects are pushed towards the poles, where they either form two nearly stationary trapped defect pairs [Figs. 1(b') and 2(b) ] or merge into two fully stationary +1 defects [Figs. 1( $\left.\mathrm{c}^{\prime}\right)$ and 2(c)]. This configuration has also been seen in the experiments of Ref. [19]. The band state strongly resembles the band found in polar systems [26]. It occurs because active particles are driven by curvature to move along geodesics, corresponding to great circles on a sphere. This effect is counterbalanced by repulsive interactions, resulting in the emergence of a finite-width band. Recent work by one of us [27] has also shown that the spontaneous emergence of bands wrapping around the equator is a generic properties of active polar fluids that arises from the interplay spontaneous flow and curvature. In our nematic system the band forms only for sufficiently long $\tau_{\text {flip }}\left(\tau_{\text {flip }} \geqslant 50\right.$ ) when the system can support long-lived local polar flows that break the symmetry. Note that such bands are indeed seen in microtubule suspensions, where the defect structure shows clear nematic symmetry. In Fig. 7 we examine the emergence of the band by looking at the evolution of the defect pair angle distribution [Fig. 7(a)] and the density profile [Fig. 7(b)] with increasing $v_{0}$. The distribution of pair angles evolves from unimodal for $v_{0}=0.1-0.2$ corresponding 

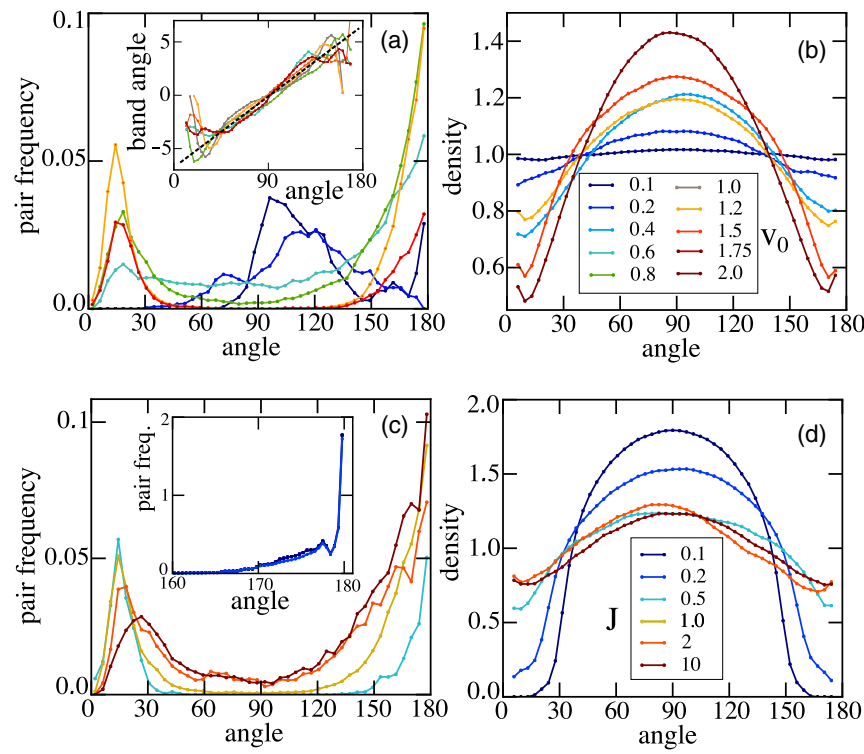

FIG. 7. Emergence of the band state as seen from the distribution of defect pair angles (a)-(c) and the density profile (b)-(d). (a) At $J=1$, with increasing $v_{0}$, the pair angle distribution evolves form unimodal with a single central peak $\left(v_{0}=0.1\right)$ to bimodal with growing peaks close to $0^{\circ}$ and $180^{\circ}\left(v_{0}=0.6-1.5\right)$. The inset shows the inclination of the direction of self-propulsion given by the director field $\mathbf{n}$ relative to the equator as a function of latitude. The black dashed line has a slope of 0.07 . (b) The corresponding density profiles evolve from homogeneous to banded. (c) For $J=0.5-10$, at $v_{0}=1.0$, the distribution peaks of the merging state shift inwards. Inset: For $J=0.1-0.2$, the two +1 defects are nearly at opposite poles. (d) In the corresponding density profile the bald spots at the poles disappear.

to a fluctuating tetrahedral arrangement of four defects smeared out by increasing diffusive motion to a bimodal one with peaks close to the location of opposing poles when the defects merge and the band state emerges. Meanwhile the density profile [Fig. 7(b)] evolves from practically uniform to a distinct peak that continuously grows with $v_{0}$, because the curvatureinduced force that drives band formation is proportional to $v_{0}$ [26]. A similar behavior is obtained by decreasing $J$ for fixed $v_{0}$ [Figs. $7(\mathrm{c})$ and $\left.7(\mathrm{~d})\right]$. For low $J=0.1-0.2$, we always observe a band state with two +1 defects [inset to Fig. 7(c)], and a very peaked density profile appears, with bald spots at the poles. At higher $J$, this gradually gives way to a nematic band with unmerged pairs of defects at the poles and the first peak position gradually shifts to larger angles and the whole distribution broadens [Fig. 7(c)]. We can explain this in part by noting that the defect core energy scales $\sim J$ when approximated by a single-constant Frank free energy [39] and so merging two $+1 / 2$ defects to form $a+1$ costs more energy at higher $J$. We see only slightly peaked density profiles for $J \geqslant 0.5$ [Fig. $7(\mathrm{~d})$ ]. The tendency of active agents to move along geodesic lines is confirmed by examining the angle between the direction $\mathbf{n}$ of self-propulsion relative to the equator [26]. The angle profile across a set of bands as a function of latitude is shown in the inset of Fig. 7(c). The slope $\approx 0.07$ for the growth of the inclination of $\mathbf{n}$ with latitude is much shallower than in the polar case for the same $J$, consistent with the suppressed appearance of nematic bands
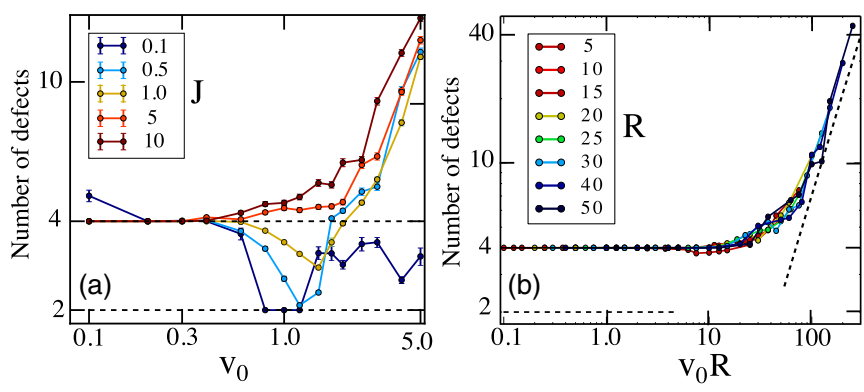

FIG. 8. (a) Number of defects of any charge as a function of $v_{0}$ and $J$ for $R=30$. At low $v_{0}$, all curves converge to the equilibrium ground state with four $+1 / 2$ defects. As the driving is increased, the low $J$ systems develop a band state with two +1 defects. At the highest driving, the systems transition to a turbulent nematic state; lower $J$ delays onset of turbulence. (b) Defect scaling as a function of sphere radius for $J=10$. The onset of turbulence scales as $v_{0} R$. The dashed diagonal line has a slope of 2 .

as compared to polar ones at high $v_{0}$. Finally, in Ref. [26] we derived an effective profile for the polar band density. We show in Appendix B that this also provides a good fit for the density profile of nematic bands.

\section{Transition to turbulence}

As even larger activity $v_{0}$, the system transitions to an active turbulent state, where pairs of $\pm 1 / 2$ defects are spontaneously created [Figs. 1( $\left.\mathrm{e}^{\prime}\right)$ and 2(e)]. In Fig. 8 we show the mean number of defects $N$ on the sphere regardless of their charges. We identify $N>4$ as the onset of turbulence.

The turbulent regime displays strong patterns of polar flow, consisting of lanes of particles moving in the same direction, as evident from Fig. 1(e). We estimate that in our confined system the transition to turbulence occurs when the spacing $l_{\alpha}$ between defects becomes comparable to the sphere radius $R$. In full hydrodynamic models in two spatial dimensions the spacing between defects in the turbulent regime has been shown to scale with the active stress as $l_{\alpha} \sim \alpha^{-1 / 2}[40,41]$. Some evidence for the same scaling has also been obtained in continuum models in the limit where viscous dissipation is negligible compared to frictional drag from the substrate [42]. In our model activity can be estimated as $|\alpha| \sim v_{0}^{2} J$, where $J$ controls the nematic stiffness and the dependence on $v_{0}^{2}$ is required by symmetry. We then estimate that the transition to turbulence will occur when we can accommodate $N$ defects of mean separation $\ell_{\alpha}$ on the surface of the sphere, i.e., $N=4 \pi R^{2} / l_{\alpha}^{2}$. This gives a prediction for the transition to turbulence as

$$
N \sim\left(R v_{0}\right)^{2} J>4
$$

This form gives a very good scaling collapse of the observed number of defects for $J=10$ where there is no band state [Fig. 8(b)].

\section{Bending state}

At low values of $J$, as already noted, the transition to turbulence is delayed. Instead, we observe an intriguing state, where the band that forms at intermediate $v_{0}$ develop a bending instability [Figs. 1(d) and 9]. This is indeed seen in the 

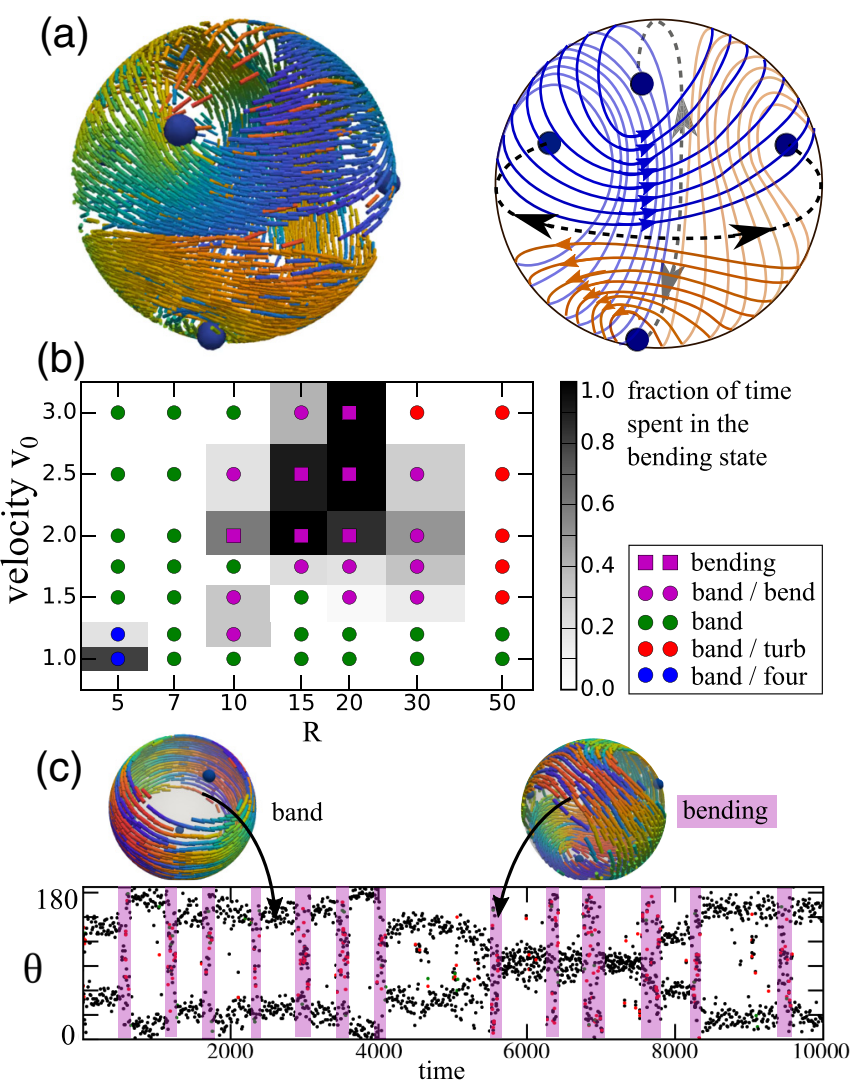

FIG. 9. Bending of the band at high activity and low alignment. High persistence lengths favor polarity sorting, leading to onedirectional polar flow. The +1 defects of the band split into pairs of $+1 / 2$ defects located at the vertices of a tetrahedron. (a) Left: Simulation snapshot at $J=0.1, v_{0}=3.0, \tau=100$. Right: Schematic drawing of the topological state. (b) Phase diagram of bending states (shading and symbols) as a function of sphere radius $R$ and $v_{0}$ at $\tau=100$; bent states exist between radii too small to allow for bending and radii large enough to allow turbulence. (c) Defect traces (polar angle $\theta$ ) for a system with intermittent dynamics for $R=20$ and $v_{0}=1.75$, band regions are separated by brief bending events (purple), snapshots correspond to the indicated time points.

experiments of Keber et al. [19] and we have also observed a similar state in the polar case [43]. In the nematic case the instability occurs via the mechanism described pictorially in Fig. 9. It is energetically favorable for the two +1 defects that sit at the poles in the band state to split into four $+1 / 2$ defects [22] because by doing so they can lower the total core energy of the configuration. The interplay between curvature, polar flow, and steric effects then drives an instability of a nature that remains to be understood. The lowest mode of oscillation of the bent band at maximum amplitude is compatible with the tetrahedral defect state of the nematic, as the large meander of the band includes one $+1 / 2$ defect in every bend, resulting in a configuration with opposing streams touching at the front and the back of the sphere, as sketched out in Fig. 9. At low $J$, the elastic energy for bending a circulating band is low, and a bending instability can be triggered by the finite size of the sphere. This occurs when the persistence length of the nematic, $\xi=v_{0} \tau_{\text {flip }}$, is comparable to the sphere circumference $2 \pi R$, so that agents can perform a full directed circulation around the sphere before reversing, resulting in lane formation and polar flows (see Appendix C). On a sphere this bending of the band may provide a generic route to the spatiotemporal chaos of the turbulent state: As shown in Fig. 9(b), bending only appears at intermediate radii and sufficiently high $v_{0}$, and is followed by a turbulent state when $R$ increases. This transition to chaos via a bending instability is also reminiscent of the results of Ngo et al. [44] for a Vicsek nematic in the plane. In the experiments, the switching between bending and band states was likewise seen only for an intermediate range of radii. Like in the experiments, the bending observed in our simulations is intermittent, as can be seen in Fig. 9(c), where we show how a system in a predominantly band state repeatedly goes through a bending transformation. For smaller $R$, runs for a given set of parameters can be locked into either state. In Appendix D, we show snapshots of the bending state for increasing $R$.

\section{CONCLUSIONS}

In this paper we presented results of detailed numerical simulations of a model of self-propelled agents with nematic alignment confined to move on the surface of a sphere. We developed algorithms for automatic detection and tracking of topological defects, which allowed us to characterize a series of emergent collective motion patterns and construct a detailed phase diagram. The onset of these patterns can be understood using ideas from liquid crystal theory, topology, and the study of active nematics in flat space. Our work not only provides a systematic framework for the experimental results of Keber et al. [19], but it additionally demonstrates that the emergent dynamical patterns of active nematics on curved geometries emerge generically as the result of the interplay of geometry and activity, and are qualitatively insensitive to the microscopic details of the model. This is substantiated by the fact that most of the states observed here have been seen in the experiment at different sphere radii $R$ and ATP concentrations. We believe that our phase diagram Fig. 4 will be especially useful. In contrast to the two existing hydrodynamic models [19,24], we can access high-activity states with large density fluctuations, which are highly experimentally relevant as bands form there.

A full continuum analytical theory of this system is lacking at present. Taking the proper hydrodynamic limit of agentbased active nematic models at high densities is complex [29]. Continuum active nematic theories rely on the presence of extensile or contractile active stresses, but the origin of such stresses is unclear in particle-based models where activity is most naturally introduced as self-propulsion. This is further complicated by the presence of nonzero Gaussian curvature that provides an additional source of nonlinearity. In order to obtain nematic behavior one has to introduce a flipping time scale $\tau_{\text {flip }}$ of the director. As we showed here, the onset of most collective motion patterns is sensitive to this time scale. Furthermore, we showed that while the behavior predicted by continuum theory emerges at large scales, there is an intermediate scale in which the nonuniversal microscopic details of the model cannot be ignored and can actually dominate the physics. An important consequence is that defect motion in self-propelled active nematics, as shown here and in Ref. [30], 
is very slow, only mildly superdiffusive, and dominated by local fluctuations. In contrast, in locally extensile systems such as Refs. [25,35] a local flow field does seem to naturally emerge. Despite these limitations, we argue that agent-based models can provide valuable insight into the behavior of active nematics.

One other hurdle to a direct comparison with experiment is that we have simulated disks, not polymers. Recent planar simulations of active polymer melts by two of us show that hairpin bends in the filaments (which are also apparent in experiment) are strongly implicated in the defect dynamics [45]. In the future, in order to address some of this questions, we aim to directly simulate active polymers with an extensile activity mechanism. Preliminary results show that defect motion and oscillations dominate the motion, but only if all locally polar parts of the dynamics are suppressed. Finally, it would be desirable to move away from the dry limit and explicitly include hydrodynamic effects.

\section{ACKNOWLEDGMENTS}

The authors would like to acknowledge many valuable discussions with Mark Bowick, Daniel L. Barton, and Prathyusha K. R. This collaboration was made possible by a travel grant from the Northern Research Partnership (NRP) Fund. R.S. acknowledge support by UK BBRSC (Grant No. BB/N009789/1) and S.H. acknowledges support by the UK BBSRC (Grant No. BB/N009150/1). M.C.M. was supported by the US National Science Foundation through awards DMR1609208 and DGE-1068780 and by the Syracuse Soft Matter Program.

\section{APPENDIX A: IDENTIFYING TOPOLOGICAL DEFECTS}

We begin by discussing the algorithm used to identify and track topological defects. The identification of the defects is not completely straightforward due to large density fluctuations that accompany some of the collective motion patterns, where one has to distinguish between a defect and a bald spot. In order to discriminate between the two, we implemented a generalized computation of the winding number. For each agent we constructed a network of all of its neighbors within a cutoff distance, i.e., the contact network. For systems with large density fluctuations it is not practical to impose a predefined cutoff, and instead we iteratively adjusted the cutoff distance until each agent had at least four neighbors. We then used the contact network to construct a polygonal tessellation of the sphere. Finally, we connected geometric centers of each of those polygons to construct the dual lattice. Compared to, e.g., a Voronoi diagram and its dual Delaunay triangulation, our method has the advantage that it generalizes to any orientable surface. As a result of this construction, each vertex of the dual lattice is in the center of a polygonal loop with the nematic director, $\mathbf{n}$, or velocity, $\mathbf{v}$, assigned to each of its corners. Each loop then acts as a discrete integration path for $\mathbf{n}$ or $\mathbf{v}$. Finally, we projected the field vectors onto the local tangent plane (plane of the polygonal loop), and integrated angle differences between two consecutive vertices in the counterclockwise direction around the contour, giving us the half-integer values of the topological charges for each loop center. In Figs. 10(a)
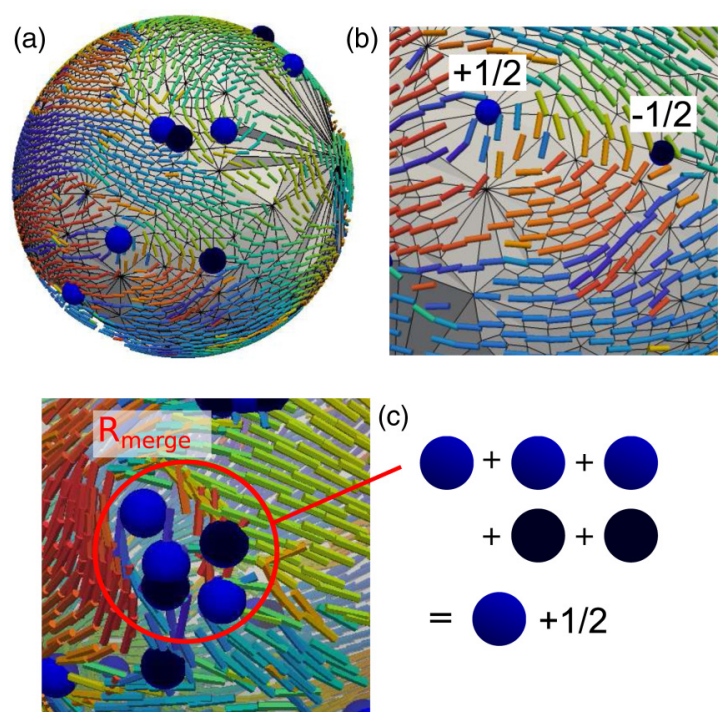

FIG. 10. (a) Example of the contact network and its dual (gray polygons) on a sphere with substantial density fluctuations. We use the same color convention for the discrete director field as in Fig. 1. (b) Zoom-in onto a pair of a positive and a negative $1 / 2$ defects. (c) Illustration of the merging technique for spots with local disorder. The five $\pm 1 / 2$ defects within $R_{\text {merge }}$ combine into one $+1 / 2$ defect.

and 10(b) we show examples of $\mathbf{n}$ fields, tessellations, and defects. Defects together with their charges are shown in Figs. 1 and 9. Determining the total number of defects allows us to easily identify the transition to turbulence (Fig. 8).

We then tracked defects between consecutive snapshots of a simulation by matching defects of a given charge with the nearest defect with the same charge in the previous frame. To reduce spurious tracking errors due to proliferation of defect clusters at spots with local disorder, we first merges all defect charges within a radius $R_{\text {merge }}=5 \sigma$, as shown in Fig. 10(c). We were able to track $+1 / 2$ defects in the four defect, merging, and bending states, and +1 defects in the band state. We did not attempt to track $-1 / 2$ defects, or defects in the turbulent state. Using a spherical coordinate system, the position of each
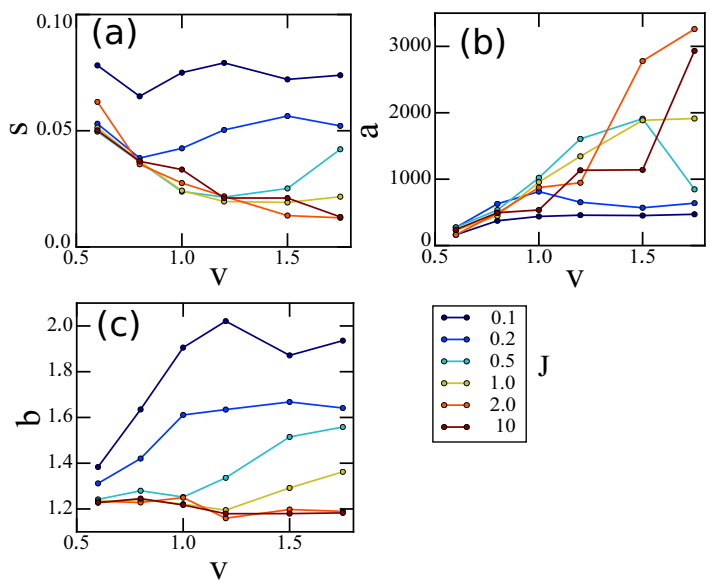

FIG. 11. Results of the fits of the angle and density profiles to the polar band theory. 


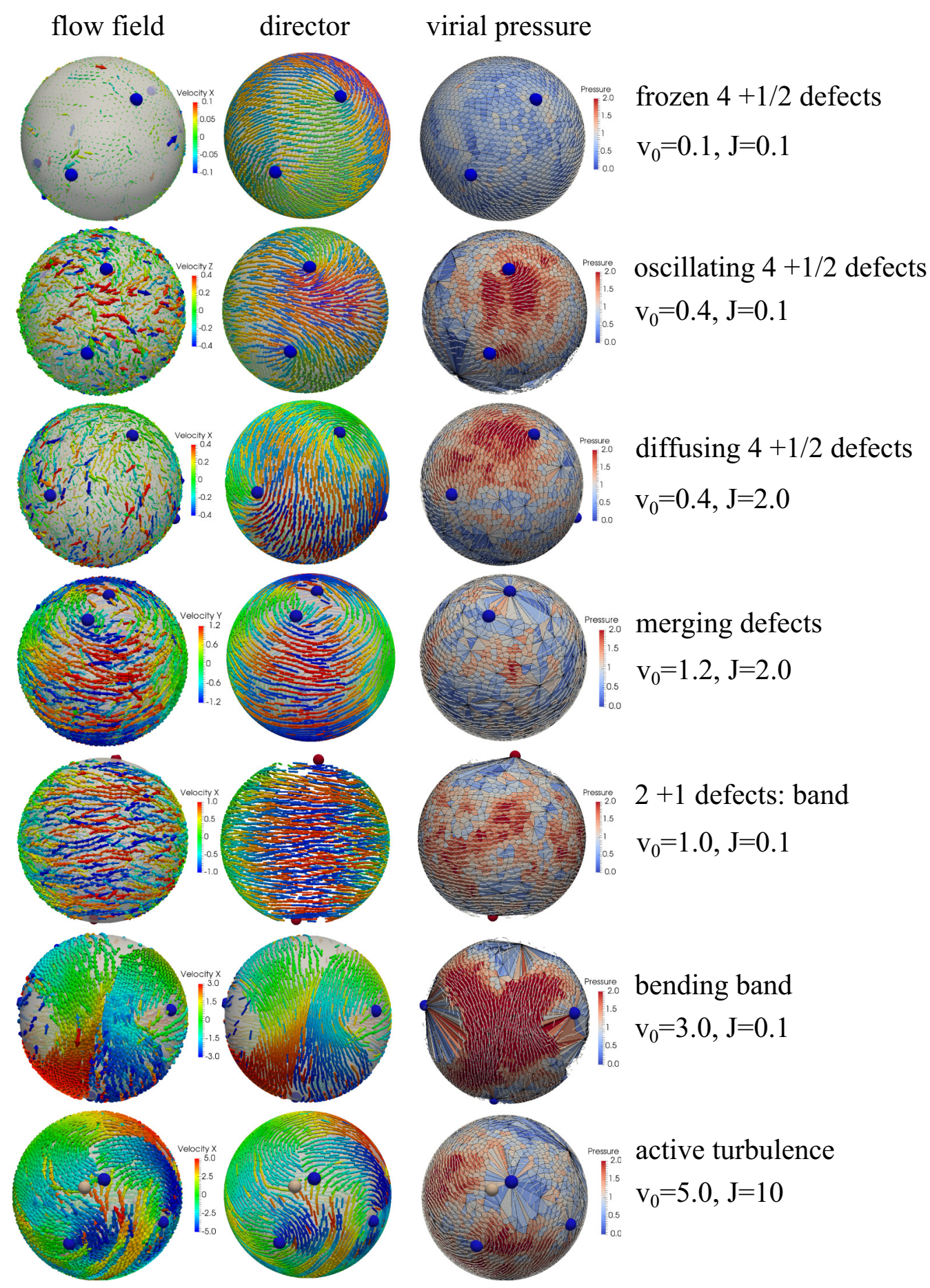

FIG. 12. Flow fields (left), nematic director fields (middle), and pressure (right) in the different dynamical patterns that we observe. Here both flow field and nematic field have been colored by the same component of motion (indicated in the caption for the velocity). The limits on the velocity color bars correspond to $\left(-v_{0}, v_{0}\right)$. For the virial pressure, we compute $p_{i}=\sum_{j} \mathbf{r}_{i j} \cdot \mathbf{F}_{i j} / A_{i}$ for each agent. Here the sum runs over the contact neighbors $j$ of $i$, and $A_{i}$ is the area of the tile belonging to $i$ computed from the dual to the contact network. On the image, each tile has been colored by its pressure. As in Fig. $1,+1 / 2$ defects are blue, $-1 / 2$ defects are tan, and +1 defects are red.

defect was identified by a unique $\theta$ and $\phi$. Figure 1 shows the stereographic projections of representative defect trajectories for the various states with the defects tracked in different colours. The corresponding trajectories in the sphere are shown in Fig. 2.

\section{APPENDIX B: BAND PROFILES}

In Ref. [25], we derived an effective equation for the band density

$$
\begin{aligned}
& \rho(\theta)=a\left[\left(\sin s \theta-\sin s \theta_{m}\right)\right]+b, \quad \theta \in\left[\theta_{m}, \pi-\theta_{m}\right] \\
& \rho(\theta)=0, \quad \text { otherwise, }
\end{aligned}
$$


where $a=\frac{1}{2 R}\left[\frac{R}{2 \sigma}\right] \frac{v_{0}}{\mu k s}$ and $b / a=\frac{2 \sigma}{R} s \cos s \theta_{m}+\frac{1}{a}$ for the polar case (note that in Ref. [25], we chose $\theta=0$ at the equator, whereas here, $\theta=\pi / 2$ at the equator). Equation (B1) describes a sine profile symmetric about the equator between two band edges located at $\theta_{m}$ and $\pi-\theta_{m}$. Here $s$ is the effective coupling strength between the active force direction $\mathbf{n}$ and the angle from the equator. It can be measured as the slope of the $\mathbf{n}$-angle profiles $\alpha(\theta)$. An example of such a profile in the full band state at $J=0.1$ is in the inset of Fig. 7(a) of the main text. The slope is clearly visible, though much shallower than in the polar case. The large deviations are in the edge regions where the density drops to zero.

In Fig. 11, we fit Eq. (B1) to the nematic bands. In Fig. 11(a), we show the $s$ from fits to the slope in the middle parts of the profiles. Like in the polar case, $s$ decreases with $J$ from a maximum of $s=0.07$ at $J=0.1$ until it reaches a plateau at $s \approx 0.03$ for $J \geqslant 0.5$. This is consistent with the scaling of the density profiles shown in Figs. 7(b)-7(d). The low values of $s$ are also consistent with the delayed appearance of the band state in the nematic system compared to the polar one. We also notice that unlike for the polar case, for the nematic case $s$ depends on $v_{0}$ : After an initial peak that coincides with the first appearance of the bands, $s$ decreases until it hits a $J$-dependent plateau value. Finally, in Figs. 11(b) and 11(c) we show the values of $a$ and $b$ extracted from fits to Eq. (B1) using the $s$ from panel a. Consistent with the polar case and the increasingly peaked density profiles that we observe, $a$ increases with $v_{0}$, though not in the linear fashion of the analytical prediction.

\section{APPENDIX C}

In Fig. 12 we show the underlying flow field and local pressure in each of the five states of the system. While in the low-activity limit, both the velocity field and the pressure are dominated by fluctuations, a coherent field with the same symmetry as the nematic texture arises at higher activity.

\section{APPENDIX D}

As shown in Fig. 9, the bending state exists only in a finite range of radii compatible with the bent geometry. In Fig. 13, we
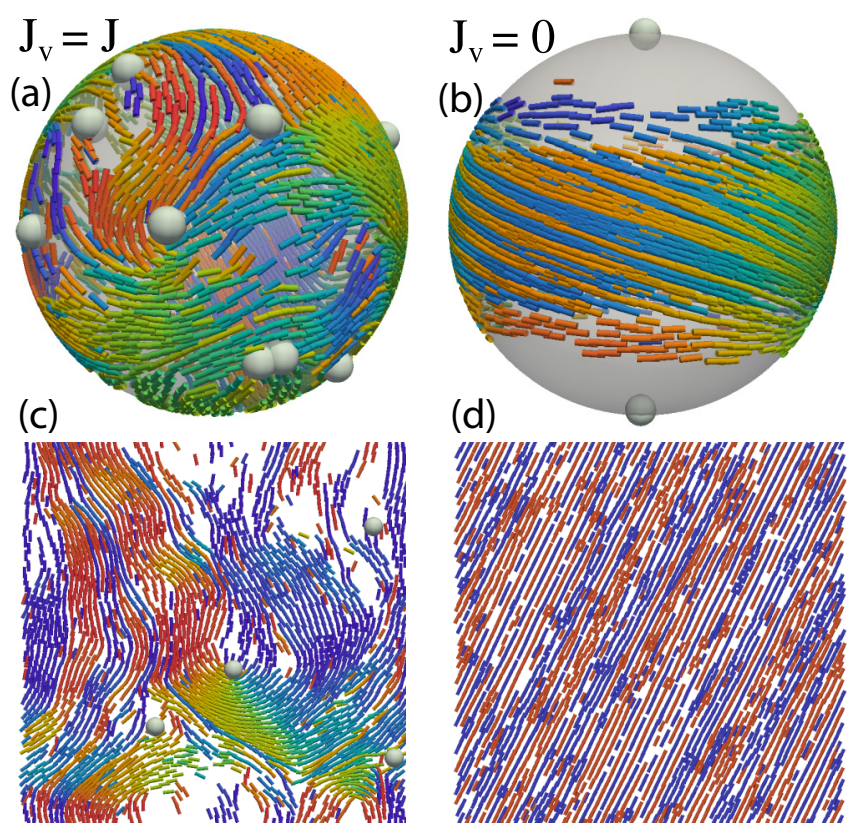

FIG. 14. Importance of the velocity coupling term $J_{v}$ in equation $1 b$. On the left, with $J_{v}=J$, as throughout the main text, both a sphere of $R=30$ (top) and a plane of $L=100$ (bottom) are turbulent for $J=1.0, v_{0}=4$, and $\tau=100$. For $J_{v}=0$ (on the right), in contrast, no pair defects nucleate from the perfectly aligned nematic state in the plane (bottom), and on the sphere, the band state persists indefinitely (top). Increasing $v_{0}$ or $J$ further does not alter this picture.

show snapshots of bending states at different radii and constant activity.

\section{APPENDIX E}

The velocity coupling term $J_{v}$ is crucial in allowing active turbulence to develop in the system, without it, no defect pairs nucleate at any activity. Figure 14 contrasts systems on the sphere and on the plane with $J_{v}=J$ (left) that display active turbulence with systems at $J_{v}=0$ (right) that do not.
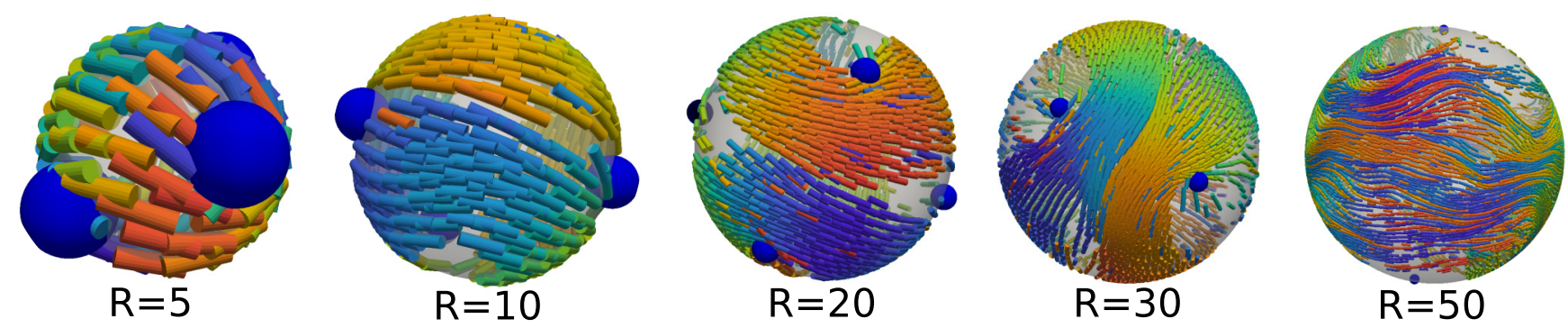

FIG. 13. Bending states as a function of system size. This is an intermittent state, which appears for only some of the simulation runs or part of a simulation run. For $\tau_{\text {flip }}=100$, the bending state disappears for $R \geqslant 50$ and for $R \leqslant 5$, we obtain a tetrahedral state instead. All systems are at $J=0.1$, and we have $v_{0}=1.0$ for $R=5$, and $v_{0}=3.0$ for $R=10-50$. 
[1] M. Marchetti, J. Joanny, S. Ramaswamy, T. Liverpool, J. Prost M. Rao, and R. A. Simha, Rev. Mod. Phys. 85, 1143 (2013).

[2] S. Ramaswamy, Annu. Rev. Condens. Matter Phys. 1, 323 (2010).

[3] T. Vicsek, A. Czirók, E. Ben-Jacob, I. Cohen, and O. Shochet, Phys. Rev. Lett. 75, 1226 (1995).

[4] R. Voituriez, J.-F. Joanny, and J. Prost, Europhys. Lett. 70, 404 (2005).

[5] R. A. Simha and S. Ramaswamy, Phys. Rev. Lett. 89, 058101 (2002).

[6] V. Narayan, S. Ramaswamy, and N. Menon, Science 317, 105 (2007).

[7] H. Chaté, F. Ginelli, and R. Montagne, Phys. Rev. Lett. 96, 180602 (2006).

[8] Y. Hatwalne, S. Ramaswamy, M. Rao, and R. A. Simha, Phys. Rev. Lett. 92, 118101 (2004).

[9] M. C. Marchetti, Nature (London) 491, 340 (2012).

[10] T. Sanchez, D. T. Chen, S. J. DeCamp, M. Heymann, and Z. Dogić, Nature (London) 491, 431 (2012).

[11] P. Guillamat, J. Ignés-Mullol, S. Shankar, M. C. Marchetti, and F. Sagués, Phys. Rev. E 94, 060602 (2016).

[12] G. Duclos, C. Erlenkämper, J.-F. Joanny, and P. Silberzan, Nat. Phys. 13, 58 (2017).

[13] K. Kawaguchi, R. Kageyama, and M. Sano, Nature (London) 545, 327 (2017).

[14] T. B. Saw, A. Doostmohammadi, V. Nier, L. Kocgozlu, S. Thampi, Y. Toyama, P. Marcq, C. T. Lim, J. M. Yeomans, and B. Ladoux, Nature (London) 544, 212 (2017).

[15] X. Yang, D. Marenduzzo, and M. C. Marchetti, Phys. Rev. E 89, 012711 (2014).

[16] Y. Fily, A. Baskaran, and M. F. Hagan, arXiv:1601.00324.

[17] T. Sato, R. G. Vries, H. J. Snippert, M. Van de Wetering, N. Barker, D. E. Stange, J. H. Van Es, A. Abo, P. Kujala, P. J. Peters et al., Nature (London) 459, 262 (2009).

[18] J. M. Collinson, L. Morris, A. I. Reid, T. Ramaesh, M. A. Keighren, J. H. Flockhart, R. E. Hill, S.-S. Tan, K. Ramaesh, B. Dhillon, and J. D. West, Dev. Dyn. 224, 432 (2002).

[19] F. C. Keber, E. Loiseau, T. Sanchez, S. J. DeCamp, L. Giomi, M. J. Bowick, M. C. Marchetti, Z. Dogic, and A. R. Bausch, Science 345, 1135 (2014).

[20] See Supplemental Material at http://link.aps.org/supplemental/ 10.1103/PhysRevE.97.042605 for movies of the motion patterns.

[21] D. R. Nelson, Nano Lett. 2, 1125 (2002).
[22] H. Shin, M. J. Bowick, and X. Xing, Phys. Rev. Lett. 101, 037802 (2008).

[23] L. Giomi, M. J. Bowick, X. Ma, and M. C. Marchetti, Phys. Rev. Lett. 110, 228101 (2013).

[24] D. Khoromskaia and G. P. Alexander, New J. Phys. 19, 103043 (2017).

[25] F. Alaimo, C. Köhler, and A. Voigt, Sci. Rep. 7, 5211 (2017).

[26] R. Sknepnek and S. Henkes, Phys. Rev. E 91, 022306 (2015).

[27] S. Shankar, M. J. Bowick, and M. C. Marchetti, Phys. Rev. X 7, 031039 (2017).

[28] S. Ehrig, J. Ferracci, R. Weinkamer, and J. W. Dunlop, Phys. Rev. E 95, 062609 (2017).

[29] T. Gao, R. Blackwell, M. A. Glaser, M. D. Betterton, and M. J. Shelley, Phys. Rev. Lett. 114, 048101 (2015).

[30] X.-q. Shi and Y.-q. Ma, Nat. Commun. 4, 3013 (2013).

[31] E. Bertin, A. Baskaran, H. Chaté, and M. C. Marchetti, Phys. Rev. E 92, 042141 (2015).

[32] M. E. Cates and J. Tailleur, Annu. Rev. Condens. Matter Phys. 6, 219 (2015).

[33] B. Szabó, G. J. Szöllösi, B. Gönci, Z. Jurányi, D. Selmeczi, and T. Vicsek, Phys. Rev. E 74, 061908 (2006).

[34] S. Henkes, Y. Fily, and M. C. Marchetti, Phys. Rev. E 84, 040301 (2011).

[35] S. J. DeCamp, G. S. Redner, A. Baskaran, M. F. Hagan, and Z. Dogic, Nat. Mater. 14, 1110 (2015).

[36] T. Frankel, The Geometry of Physics: An Introduction (Cambridge University Press, Cambridge, 2011).

[37] Y. Fily, S. Henkes, and M. C. Marchetti, Soft Matter 10, 2132 (2014).

[38] L. M. C. Janssen, A. Kaiser, and H. Löwen, Sci. Rep. 7, 5667 (2017).

[39] P. Chaikin and T. Lubensky, Principles of Condensed Matter Physics (Cambridge University Press, Cambridge, 2000).

[40] L. Giomi, Phys. Rev. X 5, 031003 (2015).

[41] E. J. Hemingway, P. Mishra, M. C. Marchetti, and S. M. Fielding, Soft Matter 12, 7943 (2016)

[42] E. Putzig, G. S. Redner, A. Baskaran, and A. Baskaran, Soft Matter 12, 3854 (2016)

[43] R. Sknepnek and S. Henkes (unpublished).

[44] S. Ngo, A. Peshkov, I. S. Aranson, E. Bertin, F. Ginelli, and H. Chaté, Phys. Rev. Lett. 113, 038302 (2014).

[45] K. R. Prathyusha, S. Henkes, and R. Sknepnek, Phys. Rev. E 97, 022606 (2018). 